Erfahrungen aus der Anwendung im Abfall- und Abwasserbereich

\section{Umweltabgaben als Innovationsimpuls}

\begin{abstract}
Nach wie vor wird kontrovers diskutiert, ob Lenkungsabgaben zur Induzierung umweltfreundlicher Innovationen geeignet sind. In der neoklassischen Umweltökonomik gelten sie als "dynamisch effizient", da sie einen permanenten Anreiz zur Suche nach umweltfreundlichen Neuerungen bieten. Dem wird z.B. von Seiten der Industrie entgegengehalten, daß statt stautlicher Eingriffe in das Preissystem vielmehr Steuersenkungen und Deregulierung erforderlich seien (1). Eine empirische Fallstudie zeigt, daß beide Positionen zu kurz greifen.
\end{abstract}

$\mathrm{H}$

Von Bodo Linscheidt inter der neoklassischen Sichtweise steht die ökonomische Grundüberlegung, daß auch und gerade die dynamische Entwicklung einer Volkswirtschaft von den relativen Preisen gesteuert wird. Durch einen geeigneten preislichen Rahmen - z.B. über eine ökologische Steuerreform - lassen sich danach die privaten Innovationsaktivitäten auf marktkonforme Weise und ohne die Notwendigkeit direkter Interventionen in eine nachhaltige Richtung lenken. Vor diesem Hintergrund hat das Finanzwissenschaftliche Forschungsinstitut an der Universität zu Köln im Rahmen eines vom BMBFT initiierten Forschungsverbundes die bisherigen Anwendungs-

Mit Öko-Effizienz zur Nachbaltigkeit?

ist das Thema des Informationsdienstes

Ökologisches
Wirtschaften 3/99

\section{Harmonisierung der} Umweltpolitik in der Europaischen Union.

\section{Das Beispiel Abfallpolitik}

ist das Thema des Informationsdienstes

\section{Ökologisches \\ Wirtschaften 4/99}

Wenn Sie potentielle Beiträge haben, wenden Sie sich bitte an die Redaktion. erfahrungen analysiert (2). Betrachtet wurden verschiedene Handlungsfelder und Akteure: erstens die Abwasser- und Abfallabgaben in der Industrie, zweitens die Abfallgebühren und ihre Innovationseffekte bei Kommunen und privaten Haushalten. Dabei wurde berücksichtigt, daß der jeweilige institutionelle Handlungskontext die durch preisliche Impulse erreichbaren Innovationen potentiell beeinflußt; im Mittelpunkt standen die fördernden und hemmenden Faktoren, von denen die Wirkungsstärke einer Abgabe unter Realbedingungen abhängt.

\section{Positiver Impuls bei allen Akteuren}

Grundsätzlich bestätigte sich dabei die neoklassische Anreizhypothese: die Verteuerung umweltschädlicher Aktivitäten wirkte bei allen betrachteten Akteursgruppen als positiver Innovationsimpuls. Abwasser- und Abfallabgaben haben in der Industrie die Anwendung verbesserter Reinigungs- und Verwertungsverfahren forciert. Auf die zum Teil drastisch gestiegenen Beseitigungskosten haben viele Kommunen mit einer verwertungsorientierten Neuorientierung ihrer Abfallwirtschaft reagiert; Kostenerhöhungen lassen sich nämlich keineswegs problemlos über höhere Gebühren an die Bürger weiterreichen, sondern setzen eine Kommune unter vergleichbaren Handlungsdruck wie ein Unternehmen. Verwertungsanreize durch verursachergerechte Gebühren haben schließlich bei den privaten Haushalten zu teilweise erstaunlichen Verhaltensänderungen gefuihrt. Die immer populärer werdende These, preisliche Abgabenanreize zerstörten die „,intrinsische Motivation“ der Haushalte $\mathrm{zu}$ freiwilligen Leistungen, wird somit nicht bestätigt (3).

\section{Die Bedeutung des Handlungsrahmens}

Gleichwohl sind die Wirkungsmechanismen in der Realität wesentlich komplexer, als es das einfache neoklassische Reaktionsmuster „Preiserhöhung $\rightarrow$ Verhaltensänderung“" unterstellt. In welchem Maße ein Preisimpuls tatsächlich zu Innovationen fuihrt, hängt von sehr vielen Faktoren ab. Während hoheitliche Preiserhöhungen in bestimmten Konstellationen einen starken Anstoß zu Innovationsaktivitäten geben, bleibt ihre Wirkung bei einem ungünstigen Handlungsrahmen gering. Als Einflußfaktoren sind u.a. zu nennen:

\section{- Informationsstand der Akteure}

Vor allem kleinere Unternehmen sind häufig über die neuesten Produktionsverfahren unzureichend informiert. Fehleinschätzungen bestehen auch bei einigen Kommunen hinsichtlich der Kosten bzw. Praktikabilität neuer Verwertungs- und Gebührensysteme. Nur bei ausreichender Information können Abgaben eine innovative Neuorientierung bewirken.

- Wabrnebmung einer Kostenerböbung als Innovationsfaktor

Je nach Unternehmensgröße, Gewinnsituation und interner Kalkulation reagieren Unternehmen auf eine Lenkungsabgabe äußerst heterogen; ähnliche Unterschiede lassen sich auch bei Kommunen feststellen. Bei privaten Haushalten entscheidet u.a. die Akzeptanz eines politischen Maßnahmenprogramms über die Reaktion auf Gebührenanreize.

- Fäbigkeit der Innovationsakteure zur Verbaltenskoordination

Im kommunalen Bereich scheitern Neuerungen häufig an politischen Rivalitäten und Konflikten. Die Fähigkeit zur Kooperation und Konsensfindung unter den Akteuren ist vielfach die entscheidende Voraussetzung für erfolgreiche Innovationen. Eine vergleichbare Rolle spielt in der Industrie die netzwerkartige Koordination von Innovationsprozessen.

Kapazitätsauslastung bestehender Entsorgungsanlagen

Emissions- bzw. abfallvermeidende Innovationen können dazu führen, daß kapitalintensive Reinigungs- bzw. Entsorgungsanlagen nicht mehr ausgelastet werden. Als starkes Innovationshemmnis haben (drohende) Überkapazitäten vor allem bei den Kommunen gewirkt: Wenn eine Müllverbrennungsanlage nicht mehr ausgelastet werden kann, sinkt das Interesse an der Abfallvermeidung und -verwertung merklich. 


\section{- Ordnungsrecht und Abgaben unterstützen sich wechselseitig}

Ein wichtiger Teil des institutionellen Handlungsrahmens ist das bestehende Ordnungsrecht. Unter statischen Effizienzaspekten wird die Wirkung von Abgaben durch diesen Rahmen häufig behindert, da Reallokationsspielräume eingeschränkt werden. Dieses Argument läßt sich jedoch auf die dynamische Perspektive nicht unmittelbar übertragen. Viele Einzelbefunde sprechen eher dafuir, daß sich Ordnungsrecht und Abgaben hinsichtlich der Innovationswirkungen wechselseitig unterstützen und verstärken: Erstens haben Auflagen praktisch immer auch abgabenähnliche Preiseffekte; so sind die ökonomischen Innovationsanreize im Siedlungsabfallbereich (gestiegene Beseitigungskosten) in erster Linie durch die Technische Anleitung Siedlungsabfall entstanden. Zweitens werden rechtliche Verhaltenspflichten und ökonomische Anreize aus Sicht der Betroffenen häufig als kumulativ-verstärkende Handlungsmotive wahrgenommen. Drittens verbessern Vollzugsprogramme den Informationsstand der Akteure und damit auch deren Fähigkeit, auf eine Kostenerhöhung innovativ zu reagieren. Viertens schließlich beschleunigen ergänzende Abgaben den Vollzug ordnungsrechtlicher Normen. Abgaben und Ordnungsrecht sind somit für die schnelle Diffusion umweltfreundlicher Neuerungen eine überaus geeignete Instrumentenkombination.

\section{- Kein Wechsel des Innovationsparadigmas}

Grundlegende Umweltinnovationen oder gar eine radikale Neuorientierung der Innovationsrichtung haben die bisherigen Lenkungsabgaben allerdings nicht in Gang setzen können; hierfür waren die Preisimpulse bei weitem zu schwach. Im wesentlichen beschränkte sich die Wirkung von Umweltabgaben auf die Diffusionsbeschleunigung bzw. die inkrementelle Weiterentwicklung bestehender Handlungsoptionen. So haben sich die Innovationswirkungen der Sonderabfallabgaben im wesentlichen auf die Forcierung der Abfallverwertung, die Wirkungen der Abwasserabgabe auf die Anwendung verbesserter Reinigungsverfahren beschränkt; für die Weiterentwicklung der prozeßintegrierten Emissions- bzw. Rückstandsvermeidung kam diesen Abgaben hingegen geringere Bedeutung zu. Durch Gebührenanreize ließ sich bei privaten Haushalten ein beachtlicher Erfolg bei der Wertstoffsammlung, kaum aber ein abfallvermeidendes Konsumverhalten erreichen.

Diese Grenzen der Steuerungsreichweite von Lenkungsabgaben lassen sich durch das aus der Evolutorischen Ökonomik stammende Konzept der pfadabhängigen Entwicklung erklären (4). Eine einmal eingeschlagene Innovationsrichtung wird danach durch eine Vielzahl ökonomischinstitutioneller Restriktionen und Rückkopplungsmechanismen - gebundenes Kapital, Skalenerträge, Lerneffekte, Netzwerkexternalitäten, eingefahrene Denkmuster oder soziale Normen stabilisiert, so daß grundlegende Neuerungen eine geringe Durchsetzungschance haben. Um eine verfestigte Entwicklung zu überwinden und neue Innovationsrichtungen anzustoßen, muß ein hoheitlicher Preisimpuls sehr ausgeprägt sein und auch die langfristigen Erwartungen wesentlich beeinflussen. Diese Anforderung haben die bisher eingeführten Abgaben nicht erfïllt.

Fraglich ist, ob der Politik die Implementierung eines derartigen „Preishebels“ überhaupt zuzutrauen ist. Diesbezüglich zeichnen sowohl die bisherigen Anwendungserfahrungen als auch die Neue Politische Ökonomie ein ernüchterndes Bild: Lenkungsabgaben mit starken Innovationsanreizen - d.h. mit hohen Abgabesätzen scheitern in aller Regel am Widerstand der betroffenen Interessengruppen. Sobald auch nur geringfügige Arbeitsplatzverluste oder Verteilungskonflikte drohen, findet der Ruf nach Ermäßigungen und Ausnahmen politisches Gehör. Wenn eine Umsetzung dennoch gelingt, sorgt die Einflußnahme der Lobbies dafür, daß die Belastungs- und damit die Lenkungswirkung gering bleibt. Ein Paradigmenwechsel ist von den dann noch verbleibenden Preisimpulsen nicht zu erwarten.

\section{Ausweg Technologieförderung?}

Vor diesem Hintergrund stellt sich die Frage, ob grundlegende umwelffreundliche Neuerungen nicht einer ergänzenden staatlichen Förderung bedürfen. Wenn die Umweltpolitik nicht zur Implementierung ausreichender Steuerungsinstrumente in der Lage ist, muß die Technologiepolitik dieses Defizit ausgleichen - zumindest dann, wenn eine nachhaltige Entwicklung wirklich angestrebt wird. Vorteil ist hierbei die höhere politische Akzeptanz: Fördermaßnahmen bieten die Möglichkeit, zusätzliche Anstoßeffekte wesentlich konfliktärmer zu realisieren, als dies durch hohe Lenkungsabgaben möglich ist. Gene- rell wird die Eignung von Subventionen als umweltpolitisches Innovationsinstrument häufig unterschätzt (5). Dies zeigen auch die bisherigen Erfahrungen. In Hessen haben z.B. gezielte Fördermaßnahmen zu abfallvermeidenden Innovationen beigetragen, die sich weder durch den Belastungsimpuls der Abfallabgabe noch durch ordnungsrechtliche Maßnahmen ergeben hätten. Derartigen Schlußfolgerungen werden regelmäßig ordnungspolitische Bedenken entgegengehalten. Befürchtet wird, daß staatliche Technologieplanung an die Stelle des freien Marktes tritt. Übersehen wird dabei, daß ein marktliches Such- und Entdeckungsverfahren nach grundlegenden Umweltinnovationen bei (zu) geringen Umweltpreisen und pfadabhängiger Entwicklung gar nicht existiert und deshalb auch nicht außer Kraft gesetzt werden kann. Neue umweltfreundliche Innovationsparadigmen - wie z.B. prozeßintegrierter Umweltschutz oder langlebiger Konsum - bilden sich nicht allein am Markt, sondern auch oder sogar nur im gesellschaftlich-wissenschaftlichen Diskurs. Eine an derartigen Paradigmen orientierte Innovationspolitik ist somit kein ordnungspolitischer Sündenfall.

\section{Anmerkungen}

(1) Bundesverband der Deutschen Industrie: Umwelistevern: Die Position der Industrie. Mehr Umwelischutz durch weniger Steuern, Köln 1995.

(2) Linscheidt, B. (Hrsg.): Umweltinnovationen durch Abgaben. Die Wirkung von Preisimpulsen im institutionellen Handlungsrahmen privater und öffentlicher Akteure, erscheint demnächst.

(3) Siehe hierzu etwa Frey, B.S./ F. Oberholzer-Gee: Zum Konflikt zwischen intrinsischer Motivation und umweltpolitischer Instrumentenwahl. In: Siebert, H. (Hrsg.): Elemente einer rationalen Umweltpolitik, Kiel. 1996, S. 207-238.

(4) Grundlegend hierzu Dosi, G.: Technological paradigms and technological trojectories. In: Research Policy, Vol. 11 (1982), S. 147-162.

(5) Vgl. Georg, S./ I. Ropke/ U. Jorgensen: Clean Technology - Innovation and Environmental Regulation. In: Environmental and Resource Economics, Vol. 2 (1992),

S. $533-550$.

\section{Der Autor}

Dr. Bodo Linscheidt ist wissenschafflicher Mitarbeiter am Finanzwissenschaftlichen Forschungsinstitut an der Universitä̀ zu Köln (Fifo).

Kontakt: Fifo, Posffach 420520, 50899 Köln, Tel. 0221/ 42-6979, Fax-2352,

E-moil: linscheidt@wiso.uni-koeln.de 
(c) 20I0 Authors; licensee IÖW and oekom verlag. This is an article distributed under the terms of the Creative Commons Attribution Non-Commercial No Derivates License (http://creativecommons.org/licenses/by-nc-nd/3.o/), which permits unrestricted use, distribution, and reproduction in any medium, provided the original work is properly cited. 